\title{
Some remarks on infinite products
}

\author{
JIM COYKENDALL, North Dakota State University, Department of \\ Mathematics, Fargo, ND 58105-5075
}

ABSTRACT: In this paper we will look at some examples of infinite product representations of elements of a commutative ring. Some obstructions to a good general theory of infinite products are explored.

\section{Introduction}

In the spirit of the recent explosion of interest in the theory of factorization, we wish to consider the possibility of the notion of infinite products. Certainly, notions of infinite products (and sums) have proved quite useful in applied mathematics and analysis as well as some branches of number theory. The usefulness of the notion of infinite products makes considering the possibility of infinite products a "moral imperative" in the setting of general commutative rings.

In [3] the present author encountered an application of infinite products in the realm of formal power series. Although the infinite product considered in [3] was mostly a formal notational convention, a similar notion of an infinite product for formal power series is studied slightly more extensively by B. Kang and M. Park in [4]. The context in which the authors of [4] and [3] utilized infinite products did not demand a full formal development of this notion (in the paper [4] the behavior of the prime spectrum of a power series ring over non-discrete valuation domains is investigated, and in [3] the dimension theory and SFT stability of power series rings over SFT rings are investigated).

The aim of this paper is to illustrate, via some examples, some possible implications of infinite products and we will show various obstructions to the development of a "clean" general theory. This work will mostly focus on examples, some proposed axioms for general infinite products, some first consequences of such a theory, and directions for further study. 


\section{A motivating example}

The paper [1] was a milestone in the study of dimension theory of formal power series rings. A classical result in the study of Krull dimension behavior in commutative rings is that if $R$ is a ring (commutative with identity) such that $\operatorname{dim}(R)=n$ then $n+1 \leq \operatorname{dim}(R[x]) \leq 2 n+1$. In [1] a near-Noetherian property termed the SFT-property (for "strong finite type") was introduced. We briefly recall that an ideal $I \subseteq R$ is SFT if there is a finitely generated ideal $B \subseteq I$ and a fixed integer $N$ such that $x^{N} \in B$ for all $x \in I$. A ring, $R$, is SFT if all its (prime) ideals have the SFT property. As it turns out, this property is central in the dimension theory of formal power series rings. In [1] it is shown that the SFT property is necessary for the dimension of $\operatorname{dim}(R[[x]])$ to be finite.

In [3] the problem of determining if the SFT property (in addition to the property that $\operatorname{dim}(R)<\infty)$ is sufficient for $\operatorname{dim}(R[[x]])$ to be finite as well as the question as to whether the SFT property is stable with respect to the adjunction of a power series variable (i.e. if $R$ is SFT then is $R[[x]]$ SFT?) are explored. These questions are both answered in the negative in [3]. In showing that the SFT property is not necessarily preserved in power series extensions, the notion of infinite products arise in a natural fashion (it should also be noted that the investigation of the prime spectra of power series rings over non-discrete valuation domains in [4], gives rise to the notion of an infinite product in a distinct, but similiar context).

As a starting point we will look at a slightly adjusted version of an example that appears in [3].

Example 2.1. Let $V$ be a one-dimensional non-discrete valuation domain with value group $\mathbb{R}$ and residue field $\mathbb{F}_{2}$. For convenience of computation we will write

$$
V:=\mathbb{F}_{2}\left[x ; \mathbb{R}^{+}\right]_{\mathfrak{M}}
$$

where $\mathbb{F}_{2}\left[x ; \mathbb{R}^{+}\right]$is the monoid ring over $\mathbb{F}_{2}$ with respect to $\mathbb{R}$ and $\mathfrak{M} \subseteq$ $\mathbb{F}_{2}\left[x ; \mathbb{R}^{+}\right]$is the maximal ideal generated by the set $\left\{x^{\alpha}\right\}_{\alpha \in \mathbb{R}^{+}}$. Let $\left\{\alpha_{i}\right\}_{i=0}^{\infty}$ be a countable collection of elements from $\mathbb{R}^{+}$. We define

$$
\prod_{i=0}^{\infty} x^{\alpha_{i}}= \begin{cases}x^{\left(\sum_{i=0}^{\infty} \alpha_{i}\right)}, & \text { if } \sum_{i=0}^{\infty} \alpha_{i} \text { converges } ; \\ 0, & \text { if } \sum_{i=0}^{\infty} \alpha_{i} \text { diverges. }\end{cases}
$$

It is easy to verify that this definition is a well-defined notion of an infinite product of any countable collection of powers of the element $x \in V$.

We briefly remark here that we are being a bit sloppy about associativity, and indeed, a more formal notion of associativity would require the notion 
of "infinite" regrouping of parentheses. What we mean by default is that given the infinite product in $R$

$$
\prod_{i=0}^{\infty} \pi_{i}
$$

there is a well-defined, deterministic way to associate an element in $R$. In this example, we associate with $\prod_{i=0}^{\infty} x_{i}^{\alpha}$, the real, positive term-series $\sum_{i=0}^{\infty} \alpha_{i}$. We will deal with associativity a bit more carefully later, but we must first make a definition.

Definition 2.2. Let $V$ be a valuation domain with value group $G$ and let $x \in V$. A subset $\left\{\alpha_{i}\right\}_{i=0}^{\infty} \subseteq G^{+}$is called admissible (with respect to $x$ ) if $x^{\alpha_{i}}$ is a well-defined element of $V$ for all $i$.

The motivation for this definition can be found in the previous example. Indeed, the element $x$ is such that $x^{\alpha} \in V$ for all $\alpha \in \mathbb{R}^{+}$, but if $u$ is a unit in $V$, then there is no guarantee of the existence of an arbitrary element of the form $(u x)^{\alpha}$. With this in mind we make a final preliminary definition.

Definition 2.3. Let $V$ be a valuation domain with value group $G$ and maximal ideal $\mathfrak{M}$. We say that the element $x \in \mathfrak{M}$ is a pseudo-uniformizer if $x^{g}$ is defined for all $g \in G^{+}$.

Basically, this definition says that if $x$ is a pseudo-uniformizer, then all subsets of $G^{+}$are admissible. In constructive examples of valuation domains, pseudo-uniformizers abound (e.g., in Example 2.1, the element $x$ is a pseudouniformizer).

We now pause to underscore a couple of observations about the behavior of the infinite product in the more general setting of (1-dimensional) valuation domains. We point out here that the "infinite product" to which the following proposition refers is the natural generalization of the infinite product that appeared in Example 2.1. That is to say, we declare (for a pseudo-uniformizer, $x$ and a subset $\left\{\alpha_{i}\right\}_{i=0}^{\infty} \subseteq G^{+}$) that

$$
\prod_{i=0}^{\infty} x^{\alpha_{i}}= \begin{cases}x^{\left(\sum_{i=0}^{\infty} \alpha_{i}\right)}, & \text { if } \sum_{i=0}^{\infty} \alpha_{i} \text { converges to an element of } G^{+} \\ 0, & \text { if } \sum_{i=0}^{\infty} \alpha_{i}=\infty \\ \text { undefined, } & \text { in all other cases. }\end{cases}
$$

Proposition 2.4. Let $V$ be a one-dimensional valuation domain with value group $G \subseteq \mathbb{R}$, valuation $v$ and maximal ideal $\mathfrak{M}$. If $x \in \mathfrak{M}$ is a pseudouniformizer, then the following properties of the infinite product $\prod_{i=0}^{\infty} x^{\alpha_{i}}$ hold. 
a) The infinite product $\prod_{i=0}^{\infty} x^{\alpha_{i}}$ is defined for all subsets $\left\{\alpha_{i}\right\}_{i=0}^{\infty} \subseteq G^{+}$ if and only if $G$ is isomorphic to either $\mathbb{Z}$ or $\mathbb{R}$.

b) If $\sigma$ is any permutation of $\mathbb{N} \bigcup\{0\}$ then $\prod_{i=0}^{\infty} x^{\alpha_{i}}=\prod_{i=0}^{\infty} x^{\alpha_{\sigma(i)}}$.

c) $\prod_{i=0}^{\infty} x^{\beta_{i}}$ divides $\prod_{i=0}^{\infty} x^{\alpha_{i}}$ if and only if $\sum_{i=0}^{\infty} \beta_{i} \leq \sum_{i=0}^{\infty} \alpha_{i}$.

d) $\prod_{i=0}^{\infty} x^{\alpha_{i}}=0$ for all subsets $\left\{\alpha_{i}\right\}_{i=0}^{\infty} \subseteq G^{+}$if and only if $V$ is discrete.

e) If $G \cong \mathbb{R}$, then any element of $\mathfrak{M}$ may be represented as a nontrivial infinite product.

Proof. We first show a) and d) in tandem. If $G$ is isomorphic to $\mathbb{Z}$, then consider the product $\prod_{i=0}^{\infty} x^{\alpha_{i}}$. The set $\left\{\alpha_{i}\right\}_{i=0}^{\infty}$ contains a least element since it is a subset of $\mathbb{N}$ and without loss of generality, we will let $\alpha_{0}$ be a least element. Clearly since every $\alpha_{i} \geq \alpha_{0}$, the series $\sum_{i=0}^{\infty} \alpha_{i}$ diverges and hence $\prod_{i=0}^{\infty} x^{\alpha_{i}}=0$. (It should also be noted that since $G \cong \mathbb{Z}$ every subset of $G^{+}$is admissible with respect to $y$ for any element $y \in \mathfrak{M}$.) If $G \cong \mathbb{R}$ then since $x$ is a pseudo-uniformizer, then $x^{\alpha}$ exists for all $\alpha \in \mathbb{R}^{+}$. In particular, the product

$$
\prod_{i=0}^{\infty} x^{\alpha_{i}}
$$

always exists; it is 0 if the series $\sum_{i=0}^{\infty} \alpha_{i}$ diverges and is $x^{\left(\sum_{i=0}^{\infty} \alpha_{i}\right)}$ if the series converges.

We now complete the proof of d). Assume that $V$ is non-discrete but that every infinite product of the form $\prod_{i=0}^{\infty} x^{\alpha_{i}}$ is 0 for all $\left\{\alpha_{i}\right\}_{i=0}^{\infty} \subseteq G^{+}$. We note that since $x$ is a pseudo-uniformizer, every subset of $G^{+}$is admissible. Note that since $V$ is non-discrete, we can inductively choose $\xi_{n} \in \mathfrak{M}$ such that

$$
v\left(\xi_{n}\right) \leq \frac{v(x)}{2^{n}} .
$$

We now let $\alpha_{0}=v(x)$ and inductively

$$
\alpha_{n}=v\left(\xi_{n}\right) \text { for all } n \geq 1 .
$$

Consider the infinite product

$$
\prod_{i=0}^{\infty} x^{\alpha_{i}}
$$

Note that since $\alpha_{n} \leq \frac{v(x)}{2^{n}}$, we have that 


$$
\sum_{i=0}^{\infty} \alpha_{i} \leq v(x) \sum_{i=0}^{\infty} 2^{-n}=2 v(x) .
$$

Hence $\sum_{i=}^{\infty} \alpha_{i}$ converges (say, to $\beta$ ). One of two things occurs at this juncture. If $\beta \in G^{+}$then $\prod_{i=0}^{\infty} x^{\alpha_{i}}=x^{\beta} \neq 0$, and if $\beta \notin G^{+}$then the infinite product $\prod_{i=0}^{\infty} x^{\alpha_{i}}$ is not defined. In either case, the contradiction finishes the proof of $\mathrm{d}$ ).

To finish the proof of a), we note that if $V$ is non-discrete and $G$ is a proper subgroup of $\mathbb{R}$ then it is well-known that the closure of $G$ (as a subset of $\mathbb{R}$ with the standard topology) is all of $\mathbb{R}$. Select an element $b \in \mathbb{R} \backslash G^{+}$and an increasing (again with respect to the ordering on $\mathbb{R}$ ) sequence $\left\{a_{n}\right\}_{n=0}^{\infty}$ of elements of $G^{+}$such that $\left\{a_{n}\right\} \longrightarrow b$. Now define $\alpha_{0}=a_{0}$ and for all $n \geq 1$, define

$$
\alpha_{n}=a_{n}-a_{n-1} .
$$

Note that each $\alpha_{n}$ is positive and since $x$ is a pseudo-uniformizer, $x^{\alpha_{n}}$ is a well-defined element of $V$ for all $n$. Now consider the infinite product

$$
\prod_{n=0}^{\infty} x^{\alpha_{n}} .
$$

Since, by construction, the infinite sum converges to an element $b \notin G$, we have our desired contradiction.

For part b), note that since each $\alpha_{i}$ is positive, if the series $\sum_{i=0}^{\infty} \alpha_{i}$ converges, then it converges absolutely. Hence any rearrangement of the series converges to the same sum. More precisely, if $\sigma$ is a permutation of $\mathbb{N} \bigcup\{0\}$ then

$$
\sum_{i=0}^{\infty} \alpha_{i}=\sum_{i=0}^{\infty} \alpha_{\sigma(i)}
$$

and hence the infinite products coincide. If the series diverges, then the same argument applies.

For part c) we first assume that $\prod_{i=0}^{\infty} x^{\beta_{i}}$ divides $\prod_{i=0}^{\infty} x^{\alpha_{i}}$ (we are tacitly assuming here that both infinite products exist). So there is a $w \in V$ such that $w \prod_{i=0}^{\infty} x^{\beta_{i}}=\prod_{i=0}^{\infty} x^{\alpha_{i}}$. Using the definition of the infinite product and computing values we get that

$$
v(w)+\sum_{i=0}^{\infty} \beta_{i}=\sum_{i=0}^{\infty} \alpha_{i}
$$


(note that if the right sum diverges, we are done and if the left diverges then so does the right). This gives that $\sum_{i=0}^{\infty} \beta_{i} \leq \sum_{i=0}^{\infty} \alpha_{i}$ (in either case, convergence or divergence).

Conversely, suppose that $\sum_{i=0}^{\infty} \beta_{i} \leq \sum_{i=0}^{\infty} \alpha_{i}$. Note that if $\sum_{i=0}^{\infty} \beta_{i}$ diverges, then so does $\sum_{i=0}^{\infty} \alpha_{i}$ and we will discard this case as degenerate (we will declare that 0 divides 0 for brevity). Assume that $\sum_{i=0}^{\infty} \beta_{i}$ converges and that its sum is $b$. If $\sum_{i=0}^{\infty} \alpha_{i}$ diverges, then we are done, so we will assume that this sum converges to $a$. Since $b \leq a$ (and $x^{b}$ and $x^{a}$ are defined since $x$ is a pseudo-uniformizer) then $x^{b}$ must divide $x^{a}$ and we are done.

Finally, for part e) assume that $m \in \mathfrak{M}$. If $m=0$, then we can represent $m$ as the infinite product $\prod_{i=0}^{\infty} x$. If $m \neq 0$ then since $G \cong \mathbb{R}$, select a positive term infinite series $\sum_{i=0}^{\infty} \alpha_{i}$ such that $\sum_{i=0}^{\infty} \alpha_{i}=\frac{v(m)}{v(x)}$ and note that

$$
\prod_{i=0}^{\infty} x^{\alpha_{i}}=x^{\left(\sum_{i=0}^{\infty} \alpha_{i}\right)}=a \in V
$$

So $v(a)=v(m)$, and hence $\frac{m}{a}$ is a unit in $V$. So we have $m=\left(\frac{m}{a}\right) \prod_{i=0}^{\infty} x^{\alpha_{i}}$ and the proof is complete.

Given an infinite product in a commutative ring with $1, R$; one can extend this product in a non-trivial way to the power series ring $R[[x]]$ as follows.

Example 2.5. Suppose that $\left\{r_{i}\right\}_{i=0}^{\infty}$ is a subset of a commutative ring with identity, $R$, such that for any subset of $S \subseteq \mathbb{N} \bigcup\{0\}$ the infinite product

$$
\prod_{i \in S} r_{i}
$$

is an element of $R$. Then the infinite product

$$
\prod_{i=0}^{\infty}\left(r_{i}+s_{i} x^{i+1}\right)
$$

where each $s_{i} \in R$ is an element of $R[[x]]$.

By infinite product, we produce a power series via the following algorithm. The constant term is the infinite product of the $r_{i}$ 's (which is well-defined by assumption). The coefficient of $x^{n}$ is determined via:

$$
\sum_{i_{1}+i_{2}+\cdots+i_{k}=n}\left(\left(s_{i_{1}} s_{i_{2}} \cdots s_{i_{k}}\right)\left(\prod_{j \neq i_{1}, i_{2}, \cdots, i_{k}} r_{j}\right)\right) .
$$

Note that any term in the sum exists since subproducts of the form $\prod_{i \in S} r_{i}$ exist. Also the sums are finite since the powers of the polynomial terms are increasing. 
This example is in the spirit of the previous example where infinite products over valuation domains are considered. In the previous example (because of absolute convergence of convergent positive term series) every subproduct of a well-defined infinite product exists. Also note that this product can be done in more generality. In particular, the terms in the successive polynomials merely have to satisfy the property that for any fixed degree, $n$, there are only finitely many polynomials of the form $r+s x^{n}$ in the infinite product.

\section{Hazards}

In the previous sections the approach to the infinite product was more informal. Basically, in all of the results, an algorithm was described to define an infinite product representation of some elements of a commutative ring with identity. In this section we will look more closely at some of the obstructions to a general notion of infinite products. In particular, for infinite products, some problems with associativity and ("infinite") commutativity naturally arise.

We will begin by defining axioms for an infinite product. All products here will be countable.

\section{Axioms for the infinite product.}

Let $\left\{r_{i}\right\}_{i=0}^{\infty}$ be a subset of a commutative ring, $R$, and assume that the infinite product $\prod_{i=0}^{\infty} r_{i}$ exists.

1. If any $r_{i}=0$ then $\prod_{i=0}^{\infty} r_{i}=0$.

2. If $R$ has an identity, then $\prod_{i=0}^{\infty} 1=1$.

3. If $\left\{n_{1}, n_{2}, \cdots\right\}$ and $\left\{m_{1}, m_{2} \cdots\right\}$ are two partitions of $\mathbb{N} \bigcup\{0\}$, then

$$
\left(\prod_{i=0}^{n_{1}} r_{i}\right)\left(\prod_{i=n_{1}+1}^{n_{2}} r_{i}\right) \cdots=\left(\prod_{i=0}^{m_{1}} r_{i}\right)\left(\prod_{i=m_{1}+1}^{m_{2}} r_{i}\right) \cdots
$$

4. Any finite rearrangement of $\prod_{i=0}^{\infty} r_{i}$ preserves the product; that is, if $\sigma$ is a permutation of $\mathbb{N} \bigcup\{0\}$ that fixes all but finitely many $r_{i}$ 's, then $\prod_{i=0}^{\infty} r_{i}=\prod_{i=0}^{\infty} r_{\sigma(i)}$.

We note the third axiom is associativity in the infinite case. One would also like for any subproduct to exist and for the last axiom to extend to arbitrary permutations (certainly one would wish for this when studying commutative rings), but we will presently see some problems with this. 
Example 3.1. Let $V$ be a 1-dimensional valuation domain with value group $\mathbb{R}$ from Example 2.1. We will show that it is impossible to extend the "natural" infinite product to the quotient field, $K$, of $V$ without incurring some strange difficulties. Indeed consider the following set of elements of $K$

$$
\left\{x, x^{\left(-\frac{1}{2}\right)}, x^{\left(\frac{1}{3}\right)}, \cdots, x^{\left((-1)^{n-1} \frac{1}{n}\right)}, \cdots\right\} .
$$

Extending the definition of infinite product to $K$ gives that $\prod_{i=1}^{\infty} x^{\left((-1)^{i-1} \frac{1}{i}\right)}=$ $x^{\ln (2)} \in V$. However, the infinite series

$$
\sum_{i=1}^{\infty}(-1)^{i-1} \frac{1}{i}
$$

is conditionally convergent. Hence, given any real number $\alpha$ (including $\alpha=$ $\pm \infty$ ), there is a rearrangement of the series that converges to $\alpha$ (one can also rearrange the series so as to diverge without making it approach $\pm \infty$ ). Hence there are rearrangements of this product that take on different values (as well as being undefined).

The previous example shows that one cannot hope to extend this infinite product in a meaningful way to powers of $x$ in the quotient field. The key was that infinite products of powers of $x$ in $V$ can be rearranged in any fashion since any infinite series taken from the positive elements of $\mathbb{R}$ either converges absolutely or diverges. This example can be extended to nondiscrete valuation domains in general.

We now relay an example that is perhaps even more disturbing and shows that the algorithm for defining an infinite product must be carefully defined.

Example 3.2. Consider $V$ to be the valuation domain from Example 2.1. We consider the infinite product (defined as in Example 2.5) in $V[[t]]$ :

$$
(x+t)\left(x^{\frac{1}{2}}+t^{2}\right)\left(x^{\frac{1}{4}}+t^{4}\right) \cdots\left(x^{\frac{1}{2^{n}}}+t^{2^{n}}\right) \cdots .
$$

As we saw in Example 2.5, this is a well-defined element of $V[[t]]$ (as written, with the algorithm supplied there). But note that

$$
\left(x^{\frac{1}{2^{n}}}+t^{2^{n}}\right)=\left(x^{\frac{1}{2^{2 n}}}+t\right)^{2^{n}} .
$$

since $\operatorname{char}(V)=2$.

If we write the infinite product

$$
(x+t)\left(x^{\frac{1}{4}}+t\right)^{2}\left(x^{\frac{1}{16}}+t\right)^{4} \cdots\left(x^{\frac{1}{2^{2 n}}}+t\right)^{2^{n}} \cdots
$$

then we see that this is not associative. In fact, grouping them in the original way gives a well-defined power series, but taking the new factors "one at a time" is not even defined since every term in the product has a linear term. 
We now produce a couple of general "obstruction" results.

Theorem 3.3. Let $R$ be a domain. If $\left\{p_{1}, p_{2}, \cdots\right\}$ are (not necessarily distinct) primes of $R$ such that the infinite product

$$
\prod_{i=1}^{\infty} p_{i}=a \in R
$$

then there is a localization of $T \supseteq R$ in which there is an infinite product of units that is a nonunit.

Proof. Let $\prod_{i=1}^{\infty} p_{i}=a \in R$ and consider the multiplicative set, $S$, generated by the set of primes $P:=\left\{p_{1}, p_{2}, \cdots\right\}$. Note that the ideal $(a) \bigcap S=\emptyset$. Indeed, if $r a=p_{1}^{m_{1}} \cdots p_{k}^{m_{k}}$ then there are two cases to consider. If there exists $p \in P$ distinct from $p_{i}, 1 \leq i \leq k$ then $p$ divides $a$ but not $p_{1}^{m_{1}} \cdots p_{k}^{m_{k}}$ which is a contradiction. If $p_{1}, p_{2}, \cdots, p_{k}$ is an exhaustive list of distinct primes from $P$, then at least one of them (say $p_{1}$ without loss of generality) must occur infinitely often as a divisor of $a$. In particular, $p_{1}^{m_{1}+1}$ divides $a$, but not $p_{1}^{m_{1}} \cdots p_{k}^{m_{k}}$.

In the localization $R_{S}$, the ideal ( $a$ ) survives (so $a$ is a nonunit), but note that in $R_{S}$ each $p_{i}$ is a unit. Hence the nonunit $a$ is an infinite product of units.

We now point out a positive result.

Proposition 3.4. Any Boolean ring admits an arbitrary, nontrivial commutative infinite product.

Proof. We first note that for an arbitrary index set, $I$, the ring

$$
\prod_{i \in I} \mathbb{F}_{2}
$$

admits a nontrivial infinite product via

$$
\prod_{j=0}^{\infty}\left\{a_{i}\right\}_{i \in I}^{(j)}=\left\{b_{i}\right\}_{i \in I}
$$

where $\left\{a_{i}\right\}_{i \in I}^{(j)} \in \prod_{i \in I} \mathbb{F}_{2}$ and

$$
b_{i}=\left\{\begin{array}{l}
1 \text { if the } i^{\text {th }} \text { term of }\left\{a_{i}\right\}^{(j)}=1 \text { for all } j \\
0 \text { otherwise. }
\end{array}\right.
$$

The fact that any Boolean ring admits a nontrivial infinite product follows from the fact that any Boolean ring is a subring of a direct product of the form $\prod_{i \in I} \mathbb{F}_{2}[2]$. 
Proposition 3.5. With the exception of $\mathbb{F}_{2}$, no field admits a nontrivial general commutative infinite product.

Proof. Certainly $\mathbb{F}_{2}$ admits an arbitrary commutative infinite product (as a special case of the previous result). So assume that $K \neq \mathbb{F}_{2}$. If $K$ admits an arbitrary infinite product, then we select an $x \in K$ such that $x$ is neither 0 nor 1. Consider the product

$$
\prod_{i=i}^{\infty} x:=y
$$

and note that $x y=y$. Since $K$ is a field and $x \neq 1, y$ must be zero. In particular, the infinite product of any nonzero, nonidentity element is 0 . We now consider the infinite product

$$
\prod_{i=i}^{\infty} x^{-1}:=z .
$$

Since we are assuming that the product is arbitrarily commutative (i.e., any rearrangement preserves an infinite product), then $y z=1$ by construction, since $\prod_{j=0}^{\infty} 1=1$. But $y=0$ and this is our contradiction.

The main theme to glean from these examples and results is that the hope of creating a general infinite product must be handled with care. Even for the fairly straightforward examples that were produced for 1-dimensional valuation domains, we saw that extending the notion of infinite products to the quotient field induced some noncommutative (albeit, "infinite" noncommutative) behavior when we allowed infinite rearrangements of infinite products. Care must also be taken to avoid problems with associativity as was pointed out in Example 3.2.

Of course, in practice, infinite products have been used in an ad-hoc way to produce results like the ones in [4] and [3], but the focus in those papers was not to create a general infinite product, but to use specific infinite product representations as a tool to prove theorems. The question remains as to the nature of general infinite products. In particular, it would be nice to classify all rings with a general commutative infinite product (of course one could also include nil rings, which are a distinct class of rings from the Boolean rings). It would also be interesting to quantify the nature of the natural noncommutativity that arises when one considers the infinite product defined earlier for the quotient field of a non-discrete valuation domain. Finally, it would be good to understand the closure of a domain with respect to an infinite product. More precisely, if $V$ is a 1 -dimensional non-discrete valuation domain with value group a proper subgroup of $\mathbb{R}$, then is the closure of $V$ with respect to our defined infinite products the larger valuation 
domain with value group $\mathbb{R}$ ? The answer is "yes" for the constructed Example 2.1. Additionally in this spirit, what is the structure of the domain obtained by adjoining all well-defined infinite products to the ring $V[[t]]$ from Example 2.5? We believe that the answers to these questions could be quite interesting and provoke interesting further directions in factorization theory.

\section{References}

[1] J. T. Arnold, Krull dimension in power series rings, Trans. Amer. Math. Soc. 177 (1973), 299-304.

[2] N. Bourbaki, Algebra I. Chapters 1-3, Translated from the French, Reprint of the 1989 English translation, Springer, Berlin, 1998.

[3] J. Coykendall, The SFT property does not imply finite dimension for power series rings, J. Algebra 256 (2002), no. 1, 85-96.

[4] B. G. Kang and M. H. Park, A localization of a power series ring over a valuation domain, J. Pure Appl. Algebra 140 (1999), no. 2, 107-124. 